\title{
Contamination of surgical mask during aerosol-producing dental treatments
}

\author{
Madline Gund $^{1}$ - Jonas Isack ${ }^{1} \cdot$ Matthias Hannig ${ }^{1} \cdot$ Sigrid Thieme-Ruffing ${ }^{2} \cdot$ Barbara Gärtner $^{2} \cdot$ Gabor Boros $^{3}$. \\ Stefan $\operatorname{Rupf}^{1}$ (D)
}

Received: 31 March 2020 / Accepted: 14 October 2020 / Published online: 27 October 2020

(C) The Author(s) 2020

\begin{abstract}
Objectives Surgical masks are usually contaminated during dental treatment. So far it has not been investigated whether a surgical mask itself can be a source of microbial transmission. The aim of this study was therefore to investigate the microbiological contamination of surgical masks during dental treatment and the transfer of microorganisms from the mask to the hands.

Materials and methods Five dental treatment modalities were studied: carious cavity preparation (P-caries, $n=10$ ), tooth substance preparation (P-tooth, $n=10$ ), trepanation and root canal treatment (P-endo, $n=10$ ), supragingival ultrasonic application (US-supra, $n=10$ ), and subgingival periodontal ultrasonic instrumentation (US-sub, $n=10$ ). Bacterial contamination of mask and gloves worn during treatment was tested by imprinting on agar plates. Additionally, before masks were tested, their outer surface was touched with a new sterile glove. This glove was also imprinted on agar. Bacteria were identified by MALDI TOF mass spectrometry. Colony-forming units (CFU) were scored: score 0: $0 \mathrm{CFU}$, score 1: $<10^{2} \mathrm{CFU}$, score 2: $>10^{2} \mathrm{CFU}$, score 3: dense microbial growth.

Results All masks and all gloves used during treatment displayed bacterial contamination (sample scores 0/1/2/3: masks 0/46/3/1 and gloves 0/31/10/9). After touching the masks with new sterile gloves, microorganisms were recovered with the following contamination scores: P-caries: 4/6/0/0, P-tooth: 2/8/0/0: P-endo: 7/3/0/0, US-supra: 0/9/1/0, US-sub: 2/8/0/0. No statistically significant differences were detected between the treatment modalities. Streptococci spp. and Staphylococci spp. representing the oral and cutaneous flora dominated.

Conclusions Surgical masks are contaminated after aerosol-producing dental treatment procedures. Used masks have a potential to be a source of bacterial contamination of the hands.

Clinical relevance Dental staff should avoid touching the outer surface of masks with their hands to prevent transmission of pathogens. It is recommendable to change the mask after each treated patient followed by hand disinfection.
\end{abstract}

Keywords Surgical face mask $\cdot$ Infection control $\cdot$ Dental practice $\cdot$ Aerosol $\cdot$ Microbiology $\cdot$ MALDI TOF mass spectrometry

Stefan Rupf

stefan.rupf@uks.eu

1 Clinic of Operative Dentistry, Periodontology and Preventive Dentistry, Saarland University, Saarland University Hospital, Kirrberger Str. 100, Building 73, 66421 Homburg/Saar, Germany

2 Institute of Medical Microbiology and Hygiene, Department of Hospital Hygiene, Saarland University, Homburg, Germany

3 Department of Oral Surgery, German Armed Forces Central Hospital, Koblenz, Germany

\section{Introduction}

Dental health care professionals are exposed to numerous risk factors [1]. Mostly important are transmissions of infectious agents such as bacteria and viruses [2], but also the exposure to solvents, nanoparticles, and other substances can occur [3].

The transfer of microorganisms does not necessarily result in a risk to dental professionals. Infection and manifestation of an associated disease depend on the pathogenicity of the bacteria, the amount of them transmitted, and the current immune status of the contaminated person $[1,4]$. Infectious agents can be transferred directly from the patient to the dental staff, from 
the dentist to the patient, and from patient to patient usually by establishing infection pathways via the staff or via (hollow) instruments, clothings, or the dental units [1, 5-7]. The highest risk for the transmission of pathogens occurs by direct blood to blood contact. However, the most common and most intensively investigated source of infection in the dental practice is aerosol. Aerosols are released in numerous dental treatment modalities [8]. These are cleaning of oral surfaces with airwater spray, preparation of carious and non-carious tooth substances with high-speed handpieces, supra- and subgingival cleaning of biofilm contaminated tooth surfaces with ultrasonic devices or powder-water spray, and endodontic and oral surgery using ultrasonic instruments [9]. These aerosols may contain microorganisms from the oral cavity or from biofilms of the dental unit, as well as blood droplets and blood-borne viruses $[1,2,6,10,11]$. The treatment of numerous patients per day exposes dental staff to a high frequency to this contaminative agent [12].

To protect staff and patients, numerous unspecific protective measures are applied. Dental units, surfaces, and instruments are disinfected and sterilized, aerosols are reduced by suction, and staff disinfect their hands and wear protective clothing and specific protective equipment. Also rinsing of the oral cavity using chlorhexidine or other disinfectants prior to treatment is a protective measure [4]. The protective equipment for dental staff consists of gloves, goggles, and surgical masks. For surgical interventions, hair cover and gowns are also used. Clear recommendations have been formulated for hand disinfection and the use of sterile or non-sterile gloves. These recommendations are currently formulated at the level of state-associated boards [13]. However, studies on the compliance with infection control practices in dentistry do not show very encouraging results. Hand disinfection is frequently not performed correctly before dental work or after removing gloves as defined by the guidelines [14].

In contrast to gloves, the correct use of face masks has so far not been a major issue in guidelines. There are only a few recommendations [15] and very few systematic studies on their correct use. Previous studies have focused mainly on the safety of dental health care workers against high-risk pathogens [16]. The contamination potential of surgical masks themselves was investigated in only one study. The authors have found microorganisms accumulate on the outer surface of the surgical mask when masks were used more than $2 \mathrm{~h}$ [17]. However, the method of usage described in this study differs fundamentally from the application in dentistry. In dentistry, (I) the surgical face mask is usually worn for shorter periods of time; (II) moreover, it is used on nearly every patient, and (III) the face mask is contaminated with microbial aerosols during almost every treatment. It has not yet been investigated if microorganisms from oral biofilms released in aerosols during dental treatment survive on the surface of face masks. Similarly, it is unclear if it is possible to transfer microorganisms from a contaminated face mask to other surfaces. The objectives of this study were therefore (I) to investigate the microbial contamination of the face mask during different aerosol-producing dental treatment modalities and (II) to analyze if touching of the contaminated face mask had a contamination potential.

\section{Materials and methods}

\section{Treatment and subjects}

Five typical dental treatment modalities in which aerosol release has to be expected were included in the study. Each of them comprised 10 consecutively included cases: high-speed/ medium-speed preparation of a carious cavity using a rubber dam (P-caries), final high-speed preparation of caries free tooth substance for an indirect restoration without rubber dam (P-tooth), trepanation of a tooth for endodontic treatment with subsequent manual chemo-mechanical root canal preparation under rubber dam, and use of 3\% sodium hypochlorite rinsing (P-endo), supragingival calculus, stain and biofilm removal with ultrasonic device (US-supra), and subgingival instrumentation with ultrasonic instruments and hand curettes (US-sub).

For the rotating instruments as well as for the ultrasonic application, dental unit water was used for cooling. Evacuation was established by means of conventional dental suction (CDS) using a cannula of $3.3 \mathrm{~mm}$ in diameter (suction flow $1.1 \mathrm{l} / \mathrm{s}$ ) and high-volume evacuation (HVE, tube of $8.0 \mathrm{~mm}$ in diameter, suction flow $6.0 \mathrm{l} / \mathrm{s}$ ). The CDS was placed lingually from the lower central incisors, even if rubber dam was used. The HVE was held by an assistant.

Dental practitioners $(n=14)$ wore sterile gloves (Gammex Latex, Ansell, Brussels, Belgium), surgical face masks (tieband medical face mask type II, Mölnlycke Health Care, Düsseldorf, Germany), and protection eyewear (Safeview eyewear, Halyard, Neunkirchen, Germany). Hygienic hand disinfection was executed before the protective equipment was applied. Direct contact with the skin, oral mucosa, and teeth of the patients was avoided. All instruments were sterile. The dental unit and the surrounding surfaces were disinfected by wiping (Celtex Wipes, Lotfex, Bremen, Germany). The room temperature was $20-22{ }^{\circ} \mathrm{C}$ with $40-60 \%$ relative humidity.

Only patients without known infectious diseases were included in the study. No individual patient or practitioners' data were recorded. All samples were anonymized. Verbal informed consent was obtained from all participants. Ethical approval for the study was obtained from the Ethics Committee of the Saarland Medical Association (Vote No. 181/19). 


\section{Sampling}

Microbiological sampling was conducted $30 \mathrm{~min}$ after affixing of the surgical mask and immediate starting of aerosol-releasing dental work. Three samples were collected from each treatment session. (I) Microbial aerosol contamination was examined on the face mask. (II) Direct contamination was tested on gloves worn during treatment. (III) Finally, an indirect path of contamination was evaluated by touching the used face mask for $5 \mathrm{~s}$ with a new sterile glove. The order of microbiological sample collection was as follows: first, glove worn during treatment (Fig. 1a), second, new sterile glove after touching the face mask (Fig. 1b), and third, face mask worn during treatment (Fig. 1c).

Unused sterile face masks and new sterile gloves $(n=5$ each) served as negative controls. Additionally, sterile face masks ( $n=15$ ) worn by three dentists for 30 min were used to control the contamination from the dentist's oral cavity. To perform these tests, the subjects were sitting alone, breathing quietly, in a ventilated clean room without speaking or coughing.

All face masks and gloves were directly imprinted for $5 \mathrm{~s}$ on brain heart infusion (BHI) agar plates (Karl Roth, Karlsruhe, Germany, diameter: 90 mm, Fig. 1 a, b). Gloves were tested directly by an imprint on the agar plate without removal of the gloves. Face masks were removed by an assisting person wearing sterile gloves without touching the surface.

\section{Culture and identification of bacteria}

All agar plates were immediately incubated at $35{ }^{\circ} \mathrm{C} \pm 2{ }^{\circ} \mathrm{C}$ with $5 \% \mathrm{CO}_{2}$ for $48 \mathrm{~h}$. Plates were read after $24 \mathrm{~h}$ and $48 \mathrm{~h}$. After $48 \mathrm{~h}$, all phenotypically different colonies were classified using matrix-assisted laser desorption/ionization time-offlight mass spectromy MALDI TOF MS (Microflex LT/SH, Bruker Daltonik, Bremen, Germany), FlexControl, and MALDI Biotyper Compass software packages (Bruker Daltonik). Colonies were picked and transferred to a stainless steel target (96-spot target, Bruker Daltonik) using a toothpick and overlayed with $2 \mu \mathrm{l}$ of matrix (alpha-cyano-4- hydroxycinnamic acid, $20 \mathrm{mg} / \mathrm{ml}$ in $0.1 \%$ trifluoroacetic acid (TFA)/acetonitrile 1:2). Samples were allowed to crystallize, washed twice with $0.1 \%$ TFA, and re-crystallized in $0.1 \%$ TFA/acetonitrile 1:2. Measurements were carried out in linear positive mode (delay $400 \mathrm{~ns}$, voltage $20 \mathrm{kV}$, mass range 2 $20 \mathrm{kDa}$ ). The spectra were externally calibrated with the standard calibrant mixture, Protein Calibration Standard I, supplied by Bruker Daltonik. Two hundred forty laser shots were applied per spot. The measurements were continued until the bacterium was clearly identified. If a spectrum could not be assigned to a known species, it was noted as "unidentified".

The colony numbers on the agar plates were classified with a scoring system. No bacterial growth was rated as score $0,1-$ 100 scattered and countable colonies (colony-forming units: CFU) as score 1, > 100 countable CFU as score 2, and agar plates displaying areas with dense microbial growth with uncountable colonies in a bacterial lawn as score 3 .

\section{Data analysis}

The qualitative and quantitative results of the bacterial contamination tests were presented descriptively. Colony scores of the samples from gloves and masks were statistically compared with the Mann-Whitney $U$ test $(p<0.05)$. The KruskalWallis test was used to compare treatment modalities $(p<0.05)$.

\section{Results}

\section{Contamination rates and scores}

All unused sterile face masks and gloves displayed no bacterial growth. In addition, the masks worn to control the infection from the dentist's oral cavity showed no contamination of their outer surface.

Bacterial contamination was detected on all 50 face masks worn during the five treatment modalities. A low bacterial growth with less than $100 \mathrm{CFU}$ (score 1) was found on 46 face masks, more than $100 \mathrm{CFU}$ (score 2) on three masks, and a dense growth with uncountable colonies (score 3 ) for one
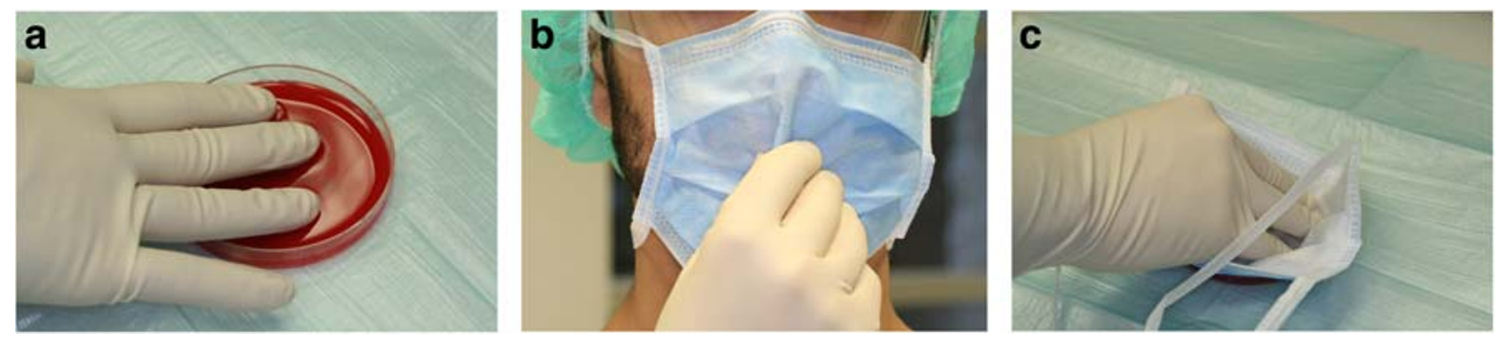

Fig. 1 Microbiological testing of gloves and face mask worn during treatment. a Direct contact sample of gloves worn during treatment, pressing three digits for $5 \mathrm{~s}$ on agar plate. b Evaluation of a contamination pathway by touching the mask for $5 \mathrm{~s}$ with a new sterile glove and imprinting this glove according to a and $\mathbf{c}$. Imprint of the mask on agar to analyze contamination with microbials containing aerosols 
mask. All 50 samples of gloves worn during treatment similarly showed bacterial contamination. Score 1 was recorded for 31 gloves, score 2 for 10 gloves, and score 3 for 9 gloves. The fresh sterile gloves, with which the face masks were touched after treatment, displayed no contamination (score 0 ) in 15 samples, score 1 in 34 samples, and score 3 in one sample. The results of the CFU scoring for the 5 treatment modalities are presented in detail in Fig. 2.

The comparison of the contamination scores revealed significantly higher numbers of colonies in gloves used during treatment compared to surgical masks $(p=0.008)$, between these gloves and the mask-touching gloves $(p<0.00001)$ as well as between masks and the gloves touching the masks $(p=0.004)$. Differences between treatment modalities were not statistically significant (treatment gloves: $p=0.7$, surgical masks: $p=0.9$, mask-touching gloves: $p=0.3$ ).

\section{Identified bacteria}

The microorganisms identified in this study are presented in Table 1. The genus Staphylococcus showed the highest prevalence. Of this genus, the species Staphylococcus epidermidis, Staphylococcus capitis, Staphylococcus hominis, Staphylococcus aureus, Staphylococcus saprophyticus, Staphylococcus warneri, Staphylococcus caprae, and Staphylococcus pettenkoferi were detected in decreasing frequency. The genus Streptococcus (Streptococcus oralis, Streptococcus mitis, Streptococcus anginosus, Streptococcus sanguinis, Streptococcus parasanguinis, Streptococcus salivarius, Streptococcus gordonii, Streptococcus infantis), Bacillus (Bacillus cereus, Bacillus circulans, Bacillus subtilis), Micrococcus (Micrococcus luteus), Rothia (Rothia dentocariosa, Rothia aeria), Neisseria (Neisseria macacae, Neisseria perflava, Neisseria subflava, Neisseria oralis), Penicillium (Penicillium chrysogenum), Actinomyces (Actinomyces oris), and Pseudomonas (Pseudomonas monteilii, Pseudomonas stutzeri) were also detected with several species. Further species (Acinetobacter pittii, Aerococcus viridans, Aspergillus versicolor, Dermabacter hominis, Enterococcus faecalis, Haemophilus parainfluenzae, Rhodococcus erythropolis, Sphingomonas sp., Streptomyces $\mathrm{sp}$.) were found sporadically. Masks and gloves which were in contact with the masks showed comparable microbial patterns, with a smaller frequency of the species. Also here Staphylococcus spp., Streptococcus spp., and Bacillus spp. dominated. Importantly, in three samples, two face masks and one treatment glove, S. aureus was detected. These strains were methicillin sensitive as shown by susceptibility testing. Concerning the bacterial species, no systematic differences were apparent between the treatment modalities.

\section{Discussion}

Two important findings were obtained through this study. First, we proved that microorganisms survive on surgical masks as used during dental work for a timeframe of at least



Fig. 2 Summary of the detection frequency and scoring of microbes on agar plates after sampling of gloves worn during treatment, surgical masks, and Mask-touching gloves. The columns each represent the 10 microbiological samples of the 5 treatment modalities carious cavity preparation (P-caries), tooth substance preparation (P-tooth), trepanation and root canal treatment (P-endo), supragingival ultrasonic application (US-supra), subgingival periodontal ultrasonic instrumentation (USsub). The columns are arranged according to the CFU scoring (score 0 : no bacterial growth; score 1: 1-100 scattered CFU; score 2: > 100 CFU; score 3: dense microbial growth). No statistically significant differences were found between the different treatment modalities (treatment glove: $p=0.7$, surgical mask: $p=0.9$, mask-touching glove: $p=0.3$ ). The contamination of surgical masks was significantly lower than of treatment gloves ( $*, p=0.008)$. Mask-touching gloves displayed significantly lower contamination than surgical masks $(* *, p=0.004)$ 
Table 1 Species of microorganisms classified within this study and frequency of their detection from gloves and masks. The typical habitat in human is indicated (oral: o, dermal: $d$, low frequency: in branches). The total number of all positive samples (max. $n=150$, bold), as well as from gloves worn during treatment, surgical masks, and glove-touching masks (max. $n=50$ each, gray), is indicated. Microbial species detected on gloves and masks are presented according to the treatment modalities (max. $n=10$ each, italics) carious cavity preparation (P-caries), tooth substance preparation (P-tooth), trepanation and root canal treatment (Pendo), supragingival ultrasonic application (US-supra), and subgingival periodontal ultrasonic instrumentation (US-sub). Colonies only identified on upper taxonomic levels are indicated as spp. or as gram positive or gram negative rods
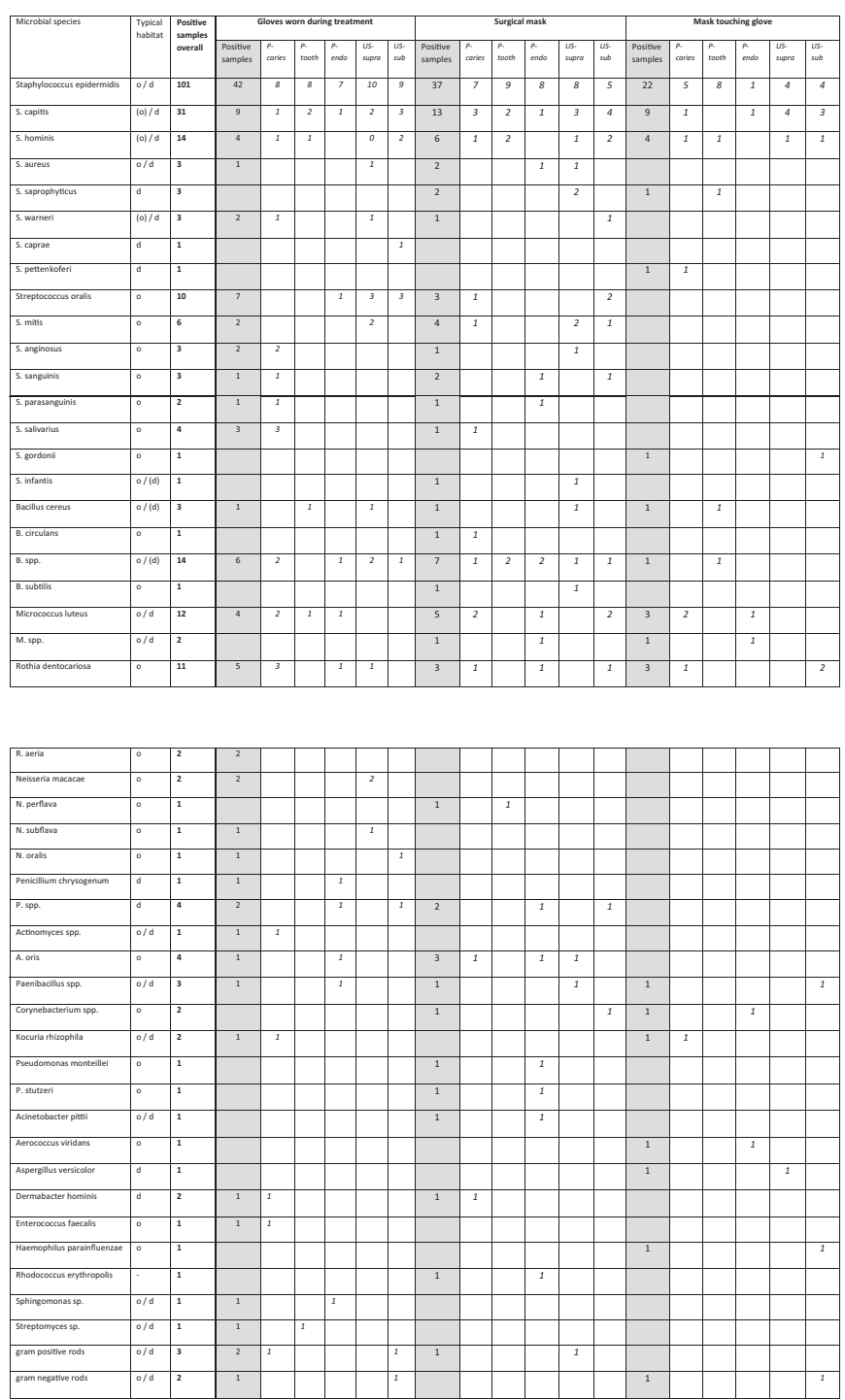

about $30 \mathrm{~min}$. Secondly, we demonstrated that viable microorganisms can be recovered by the hands from the contaminated masks and transferred to a susceptible surface. The study has thus proven a contamination pathway that has not been described so far. From the results of this study, it can be assumed that there is a risk of infection from a contaminated surgical mask via the hands to any other surfaces or to humans in dental facilities.

We showed clearly in our study that the hands could be contaminated by touching a contaminated face mask. This might result in a contamination of the dentist's hand with microorganisms from the patient treated before with a relevant risk for the next patient or the therapeutist itself. As a consequence, face masks must be discarded after each patient. In addition, the hands must be disinfected after touching of the face mask, e.g., if the mask is removed after an aerosol-producing therapeutical procedure to talk to the patient and replaced for the next therapeutical step.

Within this study, aerosol-releasing treatment techniques were applied which are used typically several times in daily dentistry. The usual measure to prevent the dissemination of 
aerosols, the high-volume evacuation, was also applied. The evacuation proved not to be able to achieve a complete prevention of contamination of the outer surface of the surgical masks. This result confirms former studies on the exposure of the dentist to fine particles or microorganisms despite using the high-volume evacuation [18]. This underlines the importance of the mask for the protection of dental staff. Remarkably, the different treatment modalities showed no significant differences in microbial contamination of the mask and the transfer of microorganisms from the mask to the sterile gloves. The surgical mask seems to provide excellent conditions for the survival of oral or dermal bacteria. This could be caused by the humidity of the exhaled air of the mask wearer. Moreover, the temperature of the exhaled air, which is almost body temperature, is perfect for the replication of many bacteria. In a previous study [17], it was found that when wearing surgical masks for more than $2 \mathrm{~h}$, an increasing number of microorganisms from the environment or from the oral cavity and respiratory system of the mask wearer accumulate. In the present study, sterile masks were worn for $30 \mathrm{~min}$. No viable microorganisms could be detected in these control experiments. Although this result is in line with another study [17], it cannot be ruled out that, in addition to the patient's own microorganisms, microorganisms from the dentist's oral cavity may also reach the outer layer of the mask through the face mask. However, this limitation is not relevant for the conclusions of this study.

The majority of the microorganisms found in our study were typical bacteria of the oral or dermal microbiome. The species with the highest prevalence in this study was S. epidermidis. It was detected on two-thirds of the studied surgical masks and gloves (Table 1). One-fifth of masks and gloves showed contamination with other Staphylococci spp. The species M. luteus, $R$. dentocariosa, S. oralis, and Bacillus spp. were each detected on more than ten masks and gloves.

Although the contamination scores for the masks and the sterile gloves that touched the masks were significantly lower than that for gloves worn during dental work, a similar microbiological spectrum was identified.

The sampling technique and microbiological methodology used in the study had strengths and limitations. One strength of the cultivation of microorganisms on BHI agar is the exclusive detection of viable microorganisms. Non-viable bacteria are not able to cause infections. The selected agar, like any other microbiological substrate, has selective properties but is usually employed to detect the vast majority of fastgrowing species. Obligate anaerobic microorganisms are not recorded, nor are various slowly growing bacteria. For MALDI TOF MS diagnostics, only colonies with different phenotypes were selected. This might led to an underestimation of the microbial spectrum. In cases of dense microbial growth, several areas were analyzed. However, competitive growth may also have reduced the detected microbial spectrum. The technique of microbiological sample collection may also underestimate contamination and microbial diversity on masks and gloves. The surgical mask is made of airpermeable material. The contact sample on agar probably only detects the microbes adhering to the surface. The gloves on the agar plates with an inner diameter of $90 \mathrm{~mm}$ could also not be completely brought into contact with the culture medium. In summary, the total numbers of viable microorganisms on both the mask and the gloves must be expected to be higher in reallife settings than in our study.

Some specific bacteria that were detected on masks and transmitted via the hands to agar plates will be discussed in the following. Each of the coagulase-negative staphylococci such as $S$. epidermidis or even $S$. aureus is potentially multiresistant bacterial species. The prevalence of $S$. aureus was low in this study and lower than found in a previous study [19]. This species was only detected in three samples. Reasons for this could be that only patients who did not report general diseases were enrolled in this study and dental staff were informed and were highly compliant with the hygienic standards in our clinic. Reports are available in the literature showing a higher prevalence of $S$. aureus in dental students than in control groups [20,21], but other studies revealed lower carriage rates [16]. Nevertheless, this species naturally represents a risk pathogen and in this study, a possible transmission path for S. aureus was demonstrated.

The most frequently isolated microorganism in this study was $S$. epidermidis. It was detected in 22 of the 50 microbiological samples obtained from gloves touching the surgical mask. This high detection frequency is in line with the results of other studies [22]. Staphylococcus epidermidis is the most common member of coagulase-negative staphylococci on human epithelial surfaces and it has to be regarded as an important nosocomial pathogen [23].

The other detected microorganisms such as S. capitis, $S$. oralis, M. luteus, or $R$. dentocariosa and all others are oral or dermal bacteria of the commensal flora. All these microorganisms are not pathogenic to healthy individuals, but may be hazardous to immunosuppressed or immunocompromised patients. Since in individual cases the health status of the patient and the risk factors for a facultative pathogenic species to become pathogenic are not always clear, it would be reasonable to consistently implement compliance with regulations and recommendations for the prevention of nosocomial infections [14]. For an infection and a clinical manifestation of a disease in a dentist or dental staff, both frequency of exposure and the virulence of the pathogen are of importance [1]. As a consequence, constant preventive behavior is of high importance since it is not possible to assess in dental practice whether a patient is colonized with pathogenic or facultative pathogenic microorganisms that can be transferred in the respective dose to a susceptible dental health care professional. 
In this study, the contamination of face masks by viruses was not investigated. This represents an additional risk for dental staff and patients. However, in the context of the COVID-19 pandemic, general hygiene measures and those aimed at preventing the transmission of microorganisms should not be compromised.

\section{Conclusion}

The results of the study confirm that surgical masks are contaminated after aerosol-producing dental treatment procedures. As a consequence, masks are a potential source of contamination of gloves or the hands when touching a used surgical mask. The mask, which is useful for personal protection, should be discarded after each patient contact. Touching the outer surface of the mask should be avoided at any time. After touching or removing the mask, the hands must be disinfected.

Acknowledgments We would like to acknowledge the dentists and students of the Clinic of Operative Dentistry, Periodontology and Preventive Dentistry and the staff of the Institute of Medical Microbiology and Hygiene of the Saarland University Hospital for their kind support.

Author contributions Conceptualization, Madline Gund, Matthias Hannig, Barbara Gärtner, Stefan Rupf.

Investigation, Jonas Isack, Madline Gund, Stefan Rupf, Sigrid Thieme-Ruffing.

Data analysis and interpretation, Jonas Isack, Gabor Boros, Sigrid Thieme-Ruffing, Madline Gund, Stefan Rupf.

Writing, Madline Gund, Stefan Rupf, Barbara Gärtner.

Project administration, Stefan Rupf, Barbara Gärtner, Matthias Hannig.

Resources, Matthias Hannig, Barbara Gärtner.

Funding Open Access funding enabled and organized by Projekt DEAL. The work was supported by internal resources of the Clinic of Operative Dentistry, Periodontology and Preventive Dentistry, Saarland University, Homburg, Germany and the Institute of Medical Microbiology and Hygiene, Department of Hospital Hygiene, Saarland University, Homburg, Germany.

\section{Compliance with ethical standards}

Conflict of interest The authors declare that they have no conflict of interest.

Ethical approval All procedures performed in studies involving human participants were in accordance with the ethical standards of the institutional and national research committee and with the 1964 Helsinki declaration and its later amendments or comparable ethical standards. Ethics approval for this study was obtained from the Ethics Committee of the Saarland Medical Association (Vote No. 181/19). According to this, the need for consent was waived, since a part of the protective equipment (outer surface of the face mask) was microbiologically tested without any reference to a person.
Informed consent For this type of study, formal consent is not required. Nevertheless, verbal informed consent was obtained from all individual participants included in the study.

Open Access This article is licensed under a Creative Commons Attribution 4.0 International License, which permits use, sharing, adaptation, distribution and reproduction in any medium or format, as long as you give appropriate credit to the original author(s) and the source, provide a link to the Creative Commons licence, and indicate if changes were made. The images or other third party material in this article are included in the article's Creative Commons licence, unless indicated otherwise in a credit line to the material. If material is not included in the article's Creative Commons licence and your intended use is not permitted by statutory regulation or exceeds the permitted use, you will need to obtain permission directly from the copyright holder. To view a copy of this licence, visit http://creativecommons.org/licenses/by/4.0/.

\section{References}

1. Volgenant CMC, de Soet JJ (2018) Cross-transmission in the dental office: does this make you ill? Curr Oral Health Rep 5:221-228

2. Harrel SK, Molinari J (2004) Aerosols and splatter in dentistry: a brief review of the literature and infection control implications. J Am Dent Assoc 135:429-437

3. Rupf S, Berger H, Buchter A, Harth V, Ong MF, Hannig M (2015) Exposure of patient and dental staff to fine and ultrafine particles from scanning spray. Clin Oral Investig 19:823-830

4. Sawhney A, Venugopal S, Babu GR, Garg A, Mathew M, Yadav M, Gupta B, Tripathi S (2015) Aerosols how dangerous they are in clinical practice. J Clin Diagn Res 9:ZC52-ZC57

5. Walker RJ, Burke FJ, Miller CH, Palenik CJ (2004) An investigation of the microbial contamination of dental unit air and water lines. Int Dent J 54:438-444

6. Kimmerle H, Wiedmann-Al-Ahmad M, Pelz K, Wittmer A, Hellwig E, Al-Ahmad A (2012) Airborne microbes in different dental environments in comparison to a public area. Arch Oral Biol 57:689-696

7. Winter S, Smith A, Lappin D, McDonagh G, Kirk B (2017) Failure of non-vacuum steam sterilization processes for dental handpieces. J Hosp Infect 97:343-347

8. Zemouri C, de Soet H, Crielaard W, Laheij A (2017) A scoping review on bio-aerosols in healthcare and the dental environment. PLoS One 12:e178007

9. Kedjarune U, Kukiattrakoon B, Yapong B, Chowanadisai S, Leggat P (2000) Bacterial aerosols in the dental clinic: effect of time, position and type of treatment. Int Dent J 50:103-107

10. Bennett AM, Fulford MR, Walker JT, Bradshaw DJ, Martin MV, Marsh PD (2000) Microbial aerosols in general dental practice. Br Dent J 189:664-667

11. Decraene V, Ready D, Pratten J, Wilson M (2008) Air-borne microbial contamination of surfaces in a UK dental clinic. J Gen Appl Microbiol 54:195-203

12. Garus-Pakowska A, Górajski M, Szatko F (2017) Knowledge and attitudes of dentists with respect to the risks of blood-borne pathogens-a cross-sectional study in Poland. Int J Environ Res Public Health 14(1). pii: E69

13. Kramer A, Briesch H, Christiansen B, Löffler H, Perlitz C, Reichard C (2016) Händehygiene in Einrichtungen des Gesundheitswesens. Empfehlung der Kommission für Krankenhaushygiene und Infektionsprävention (KRINKO) beim Robert Koch-Institut (RKI). Bundesgesundheitsblatt 59:1189_ 1220 
14. Mutters N, Hägele U, Hagenfeld D, Hellwig E, Frank U (2014) Compliance with infection control practices in an university hospital dental clinic. GMS Hygiene and Infection Control 9:Doc18

15. Exner M, Popp W, Nußbaum B, Jurreit A, Loczenski B, Bauer S, Birk-Hansen A, Bitterwolf K, Bobrich I, Dogru-Wiegand S, Eckardt M, Gerber H, Greef E, Häupler U, Jonas D, La Rocha V, Knoche J, Kühner-Feldes F, Poldrack R, Schaff T, Schmidt R, Steuer W (2016) Kleidung und Schutzausrüstung für Pflegeberufe aus hygienischer Sicht. Hyg Med 41:7-8

16. Petti S, Kakisina N, Volgenant CM, Messano GA, Barbato E, Passariello C, de Soet JJ (2015) Low methicillin-resistant Staphylococcus aureus carriage rate among Italian dental students. Am J Infect Control 43:e89-e91

17. Zhiqing L, Yongyun C, Wenxiang C, Mengning Y, Yuanqing M, Zhenan Z, Haishan W, Jie Z, Kerong D, Huiwu L, Fengxiang L, Zanjing Z (2018) Surgical masks as source of bacterial contamination during operative procedures. J Orthop Translat 14:57-62

18. Desarda H, Gurav A, Dharmadhikari C, Shete A, Gaikwad S (2014) Efficacy of high-volume evacuator in aerosol reduction: truth or myth? A clinical and microbiological study. J Dent Res Dent Clin Dent Prospects 8:176-179
19. Koukos G, Sakellari D, Arsenakis M, Tsalikis L, Slini T, Konstantinidis A (2015) Prevalence of Staphylococcus aureus and methicillin resistant Staphylococcus aureus (MRSA) in the oral cavity. Arch Oral Biol 60:1410-1415

20. Roberts MC, Soge OO, Horst JA, Ly KA, Milgrom P (2011) Methicillin-resistant Staphylococcus aureus from dental school clinic surfaces and students. Am J Infect Control 39:628-632

21. Martínez-Ruíz FJ, Carrillo-Espíndola TY, Bustos-Martínez J, Hamdan-Partida A, Sánchez-Pérez L, Acosta-Gío AE (2014) Higher prevalence of methicillin-resistant Staphylococcus aureus among dental students. J Hosp Infect 86:216-218

22. Al Maghlouth A, Al Yousef Y, Al Bagieh N (2004) Qualitative and quantitative analysis of bacterial aerosols. J Contemp Dent Pract 5: 91-100

23. Otto M (2014) Staphylococcus epidermidis pathogenesis. Methods Mol Biol 1106:17-31

Publisher's note Springer Nature remains neutral with regard to jurisdictional claims in published maps and institutional affiliations. 